18,12

\title{
О декорировании зигзагообразных краев наноленты эпитаксиального графена
}

\author{
(C) С.Ю. Давыдов
}

Физико-технический институт им. А.Ф. Иофрфе РАН, Санкт-Петербург, Россия

E-mail: Sergei_Davydov@mail.ru

(Поступила в Редакцию 8 октября 2018 г.)

Предложена двухцепочечная модель наноленты эпитаксиального графена, зигзагообразные края которой декорированы чужеродными адчастицами. В качестве подложки рассматривается металл. Получены аналитические выражения для функций Грина адатомов углерода и адчастиц. Для свободного состояния определен зонный спектр и предложена аппроксимация плотностей состояний. Приведены аналитические выражения для чисел заполнения в режиме сильной связи адсорбционного комплекса с подложкой. Рассмотрена также декорированная адчастицами цепочка адатомов углерода - эпикарбин.

DOI: $10.21883 /$ FTT.2019.03.47259.273

\section{1. Введение}

Декорирование графеновых образований путем присоединения к их краям чужеродных частиц (атомов, молекул, функциональных групп) продиктовано желанием управлять характеристиками таких объектов. В соответствующих теоретических работах рассматриваются наноленты [1,2], квадратные и треугольные островки [3]. Сходные во многом задачи возникали ранее при изучении кромок графита [4-6] и контактов латеральных графеноподобных структур [7-10]. Во всех цитированных работах отмечалось, что особый интерес представляют зигзагообразные кромки, электронный спектр которых характеризуется пиком плотности состояний вблизи точки Дирака. В этих работах, однако, рассматривались только свободные графеновые образования, хотя условием их реального существования в приборных структурах является наличие подложки. В настоящей работе мы рассмотрим декорирование наноленты эпитаксиального графена (эпиграфена), сформированного на металлической подложке. Поэтому в дальнейшем мы будем говорить об адатомах углерода графена и адчастицах. Для построения модели графеновой наноленты воспользуемся двумерной решеткой типа кирпичной стенки $[11,12]$, топологически эквивалентной решетке графена. Отметим, что подобный подход уже использовался нами для построения моделей декорирования кромки полубесконечного листа графена [13] и гетероконтакта латеральных структур [14]. Основной целью настоящей работы является оценка перехода заряда между адатомами графена, адчастицами и металлической подложкой, причем, в отличие от $[13,14]$ основное внимание уделяется режиму сильной связи адсорбированного комплекса с субстратом. Рассмотрена также задача о декорировании цепочки адатомов углерода (эпикарбина).

\section{2. Функции Грина и зонная структура}

Модель декорированной наноленты представлена на рис. 1. Имеем, таким образом, две сцепленные цепочки адатомов углерода с присоединенными к ним чужеродными адчастицами. Адчастицы связаны с каждым вторым адатомом края эпиграфена, что отвечает декорированию зигзагообразной кромки. Изолированным адатому углерода и адчастице припишем соответственно затравочные функции Грина

$$
g=(\omega+i \Gamma)^{-1}, \quad g_{a}=\left(\omega-\varepsilon_{a}+i \Gamma_{a}\right)^{-1}
$$

где энергия $p$-состояния адатома углерода принята за нуль, $\varepsilon_{a}$ - энергия уровня адчастицы, Г и $\Gamma_{a}-$ полуширины квазиуровней адатома углерода и адчастицы $[15,16]$. Воспользовавшись нумерацией адатомов и адчастиц $(m, n)$, представленной на рис. 1 , и считая энергию перехода между ближайшими соседями (NN.) в графене равной $t$, а между атомами графена и частицами

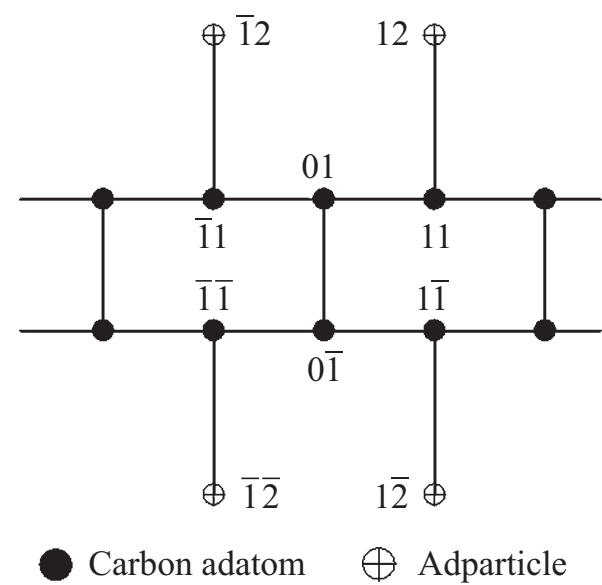

Рис. 1. Модель декорированной наноленты и нумерация атомов графена и декорирующих частиц. 
равной $\tau \tilde{t}$, запишем цепочку уравнений Дайсона для функций Грина $G_{m n, k l}[15,16]$

$$
\begin{gathered}
G_{11,12}=g \tilde{t} G_{12,12}+g t\left(G_{01,12}+G_{21,12}\right), \\
G_{12,12}\left(1-g g_{a} \tilde{t}^{2}\right)=g_{a}+g g_{a} t \tilde{t}\left(G_{01,12}+G_{21,12}\right), \\
\left(G_{01,12}+G_{21,12}\right)\left(1-\frac{g^{2} t^{2}}{1-\frac{g^{2} t^{2} \Phi(k)}{1-g g_{a} \tilde{t}^{2}}}\right)=g t \Phi(k) G_{11,12},
\end{gathered}
$$

где $\Phi(k)=4 \cos ^{2}(k a), a-$ расстояние между $\mathrm{NN}$ графена, $k$ - волновой вектор, $|k| \leq \pi / 2$.Тогда получим

$$
\begin{aligned}
& G_{01,01}(\omega, k) \equiv G_{0}(\omega, k) \\
& \quad=g\left(1-\frac{g^{2} t^{2} \Phi(k)}{1-g g a \tilde{t}^{2}}-\frac{g^{2} t^{2}}{1-\frac{g^{2} t^{2} \Phi(k)}{1-g g_{a} t^{2}}}\right)^{-1} .
\end{aligned}
$$

Аналогичным образом определим функции Грина

$$
\begin{aligned}
& G_{11,11}(\omega, k) \equiv G_{1}(\omega, k) \\
& =g\left(1-g g_{a} \tilde{t}^{2}-\frac{g^{2} t^{2} \Phi(k)}{1-\frac{g^{2} t^{2}}{1-\frac{g^{2} t^{2} \Phi(k)}{1-g_{a} \tilde{t}^{2}}}}\right)^{-1}, \\
& G_{12,12}(\omega, k) \equiv G_{a}(\omega, k) \\
& =g_{a}\left(1-\frac{g g_{a} \tilde{t}^{2}}{1-\frac{g^{2} t^{2} \Phi(k)}{1-\frac{g^{2} t^{2}}{1-\frac{g^{2} t^{2} \Phi(k)}{1-g g_{a} t^{2}}}}}\right)^{-1},
\end{aligned}
$$

Рассмотрим зонную структуру системы нанолента декорирующие частицы в отсутствии подложки, для чего положим $\Gamma=\Gamma_{a}=s \equiv 0^{+}$. Полюса функций Грина (2)-(4), определяющие дисперсию электронов, являются корнями уравнения

$$
D(\omega, k)=D_{+}(\omega, k) D_{-}(\omega, k)=0,
$$

где

$$
\begin{aligned}
D_{ \pm}(\omega, k)= & \left(\omega-\varepsilon_{a}\right)\left[\omega^{2}-t^{2} \Phi(k)\right] \\
& -\omega \tilde{t}^{2} \pm t\left[\omega\left(\omega-\varepsilon_{a}\right)-\tilde{t}^{2}\right] .
\end{aligned}
$$

Легко видеть, что уравнение (5) инвариантно относительно одновременной замены $\omega$ на $-\omega$ и $\varepsilon_{a}$ на $-\varepsilon_{a}$. На границе зоны Бриллюэна (при $|k|=\pi / 2$ и $\Phi(k)=0)$
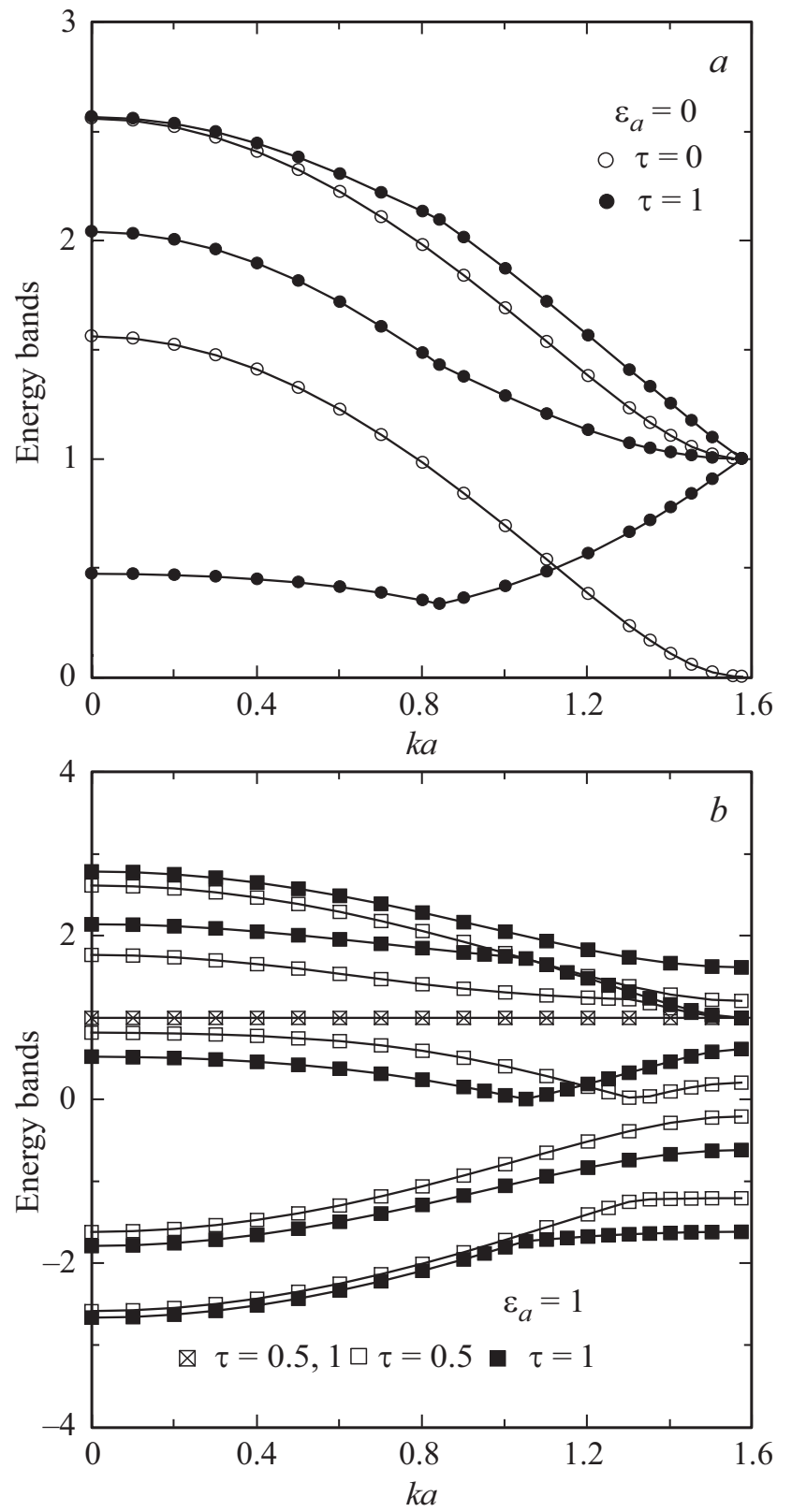

Рис. 2. Зонная структура недекорированной (a) и декорированной $(b)$ наноленты. Энергии зон приведены в ед. t. $a): \varepsilon_{a}=0, \tau=0$ (светлые кружки), $\tau=1$ (темные кружки). Вследствие симметрии относительно $\omega=0$ изображены только зоны, отвечающие положительной энергии. $b): \varepsilon_{a}=1$, $\tau=0.5$ (светлые квадраты), $\tau=1$ (темные квадраты). Бездисперсионная зона $\omega(k)=\varepsilon_{a}=1$, общая для случаев $\tau=0.5$ и $\tau=1$, обозначена квадратами с встроенными косыми крестами.

имеем $\omega= \pm t$ и $\omega=\left[\varepsilon_{a} \pm \sqrt{\varepsilon_{a}^{2}+4 \tilde{t}^{2}}\right] / 2$ (дважды вырожденный корень). Рассмотрим некоторые характерные частные случаи.

При $\tau \equiv \tilde{t} / t=0$ из (7) получаем $\omega=\varepsilon_{a}$, что отвечает уровню изолированной частицы, и зоны

$$
\omega(k)= \pm t[1 \pm \sqrt{1+4 \Phi(k)}] / 2 .
$$


Для положительных значений энергии эти зоны, отвечающие простой модели недекорированной свободной наноленты, изображены на рис. 2, a светлыми кружками. При этом на границе зоны Бриллюэна $\omega(k) \propto$ const $\pm\left(k^{\prime} a\right)^{2}$, где $k^{\prime}=(\pi / 2 a)-k$. При $\varepsilon_{a}=0$ и $\tau=1$ зоны $\omega(k)$ существенно изменяется: так, например, низкоэнергетическая ветвь $\omega(k)$ приобретает немонотонный характер с экстремумами при $k a \approx 0.84$. При этом в спектре появляется щель $\Delta \approx 2 t / 3$. Те же зонные особенности в низкоэнергетической области уже отмечались другими авторами: см., например, fig. 3 в [1], fig. 4 в [3], fig. 2 в [4], figs. 1 в[5] и [6].

Далее, положив $\omega=t+\omega^{\prime}$, где $\left|\omega^{\prime}\right| / \ll t$, легко показать, что при $\tau=1$ и $k a \rightarrow \pi / 2$ для верхней и нижней ветвей спектра имеем $\omega_{1,2}^{\prime}=\sqrt{\Phi / 2} \propto k^{\prime} a$, для средней ветви получим $\omega_{2}^{\prime} / t=\Phi / 4 \propto\left(k^{\prime} a\right)^{2}$ (здесь и далее мы нумеруем зоны сверху вниз по шкале энергии). В принципе, случай $\varepsilon_{a}=0, \tau=1$ можно также рассматривать в качестве модели свободной и не декорированной чужеродными частицами наноленты. Здесь краевой атом (типа $-11,11,-1-1,1-1)$ характеризуется двумя оборванными связями, в отличие от случая $\varepsilon_{a}=0$, $\tau=0$, где краевой атом (типа $-12,12,-1-2,1-2$ ) имеет одну оборванную связь,

Более общий случай для $\varepsilon_{a}=t, \tau=0.5$ и 1 представлен на рис. 2, $b$. При этом симметрия зон, отвечающих положительным и отрицательным энергиям, нарушается. При переходе от центра зоны Бриллюэна к ее границе наблюдается уплощение зависимостей $\omega(k)$, что также отмечалось ранее [1,3-6]. Имеется и бездисперсионная зона $\omega=\varepsilon_{a}=t$ как для $\tau=0.5$, так и для $\tau=1$. Вариация значения $\tau$ в наибольшей степени проявляется в области границы зоны Бриллюэна. Подчеркнем, что главное отличие полученных здесь зон от зон работы [13] состоит в немонотонной зависимости $\omega(k)$ для некоторых ветвей спектра, т.е. наличии экстремумов у функций $\omega(k)$.

\section{3. Свободная декорированная нанолента графена}

Для нахождения энергетической плотности состояний необходимо вычислить зависящие только от энергии функции Грина вида

$$
G_{j}(\omega)=\frac{a}{\pi} \int_{-\pi / 2 a}^{\pi / 2 a} G_{j}(\omega, k) d k,
$$

где $j=0,1$ и $a$. Начнем со свободной системы нанолента - адчастицы. При этом функции Грина (2)-(4) удобно представить в виде

$$
G_{j}(\omega, k)=C_{j}(\omega, k) / D(\omega, k),
$$

где

$$
\begin{aligned}
& C_{0}(\omega, k)= {\left[\omega\left(\omega-\varepsilon_{a}\right)-\tilde{t}^{2}\right] } \\
& \times\left[\left(\omega^{2}-t^{2} \Phi(k)\right)\left(\omega-\varepsilon_{a}\right)-\omega \tilde{t}^{2}\right], \\
& C_{1}(\omega, k)=\left(\omega-\varepsilon_{a}\right)\left\{\left(\omega^{2}-t^{2}\right)\left[\omega\left(\omega-\varepsilon_{a}\right)-\tilde{t}^{2}\right]\right. \\
&\left.-\omega\left(\omega-\varepsilon_{a}\right) t^{2} \Phi(k)\right\}, \\
& C_{a}(\omega, k)=\left(\omega^{2}-t^{2} \Phi(k)\right)\left[\left(\omega^{2}-t^{2}\right)\left(\omega-\varepsilon_{a}\right)-\omega \tilde{t}^{2}\right] \\
&-\omega t^{2}\left[\omega\left(\omega-\varepsilon_{a}\right)-\tilde{t}^{2}\right]
\end{aligned}
$$

и $D(\omega, k)$ дается выражениями (7) и (8). Так как структура функций Грина (9) сложна, получить аналитические выражения для $\rho_{i}(\omega)$ не представляется возможным. Прибегнем поэтому к следующим упрощениям. Представим функции Грина (9) в виде

$$
\begin{gathered}
G_{j}(\omega, k)=\frac{1}{2}\left[G_{j}^{-}(\omega, k)+G_{j}^{-}(\omega, k)\right], \\
G_{j}^{ \pm}(\omega, k)=\frac{c_{j}(\omega, k)}{D_{ \pm}(\omega, k)},
\end{gathered}
$$

где $\quad c_{j}(\omega, k)=2 C_{j}(\omega, k) / C^{*}(\omega, k) \quad$ и $\quad C^{*}(\omega, k)=$ $=\left[D_{+}(\omega, k)+D_{-}(\omega, k)\right]$. Из выражений (6) и (10) получим $C_{0}(\omega)=\omega\left(\omega-\varepsilon_{a}\right)-\tilde{t}^{2}$. Независимость этого коэффициента от $k$ позволяет получить аналитическое выражение для функции Грина $G_{0}(\omega)$ (см. ниже). $\mathrm{C}$ коэффициентами $c_{1}(\omega, k)$ и $c_{a}(\omega, k)$ дело обстоит сложнее вследствие их зависимости от $k$. Поэтому положим для простоты $c_{1, a}(\omega, k)=c_{1, a}^{0}(\omega)=$ $=2 C_{1, a}^{0}(\omega) /\left(C^{*}\right)^{0}(\omega)$, где верхний индекс „, $0^{\circ}$ означает, что коэффициенты $C_{1, a}(\omega, k)$ и $C^{*}(\omega, k)$ вычислены при $\Phi(k)=0$. Тогда получим $c_{1}^{0}=\left(\omega-\varepsilon_{a}\right)\left(\omega^{2}-t^{2}\right) / \omega$ и $c_{a}^{0}(\omega)=\omega^{2}-t^{2}$. Такое приближение, которое в [14] называется низкоэнергетическим, представляются вполне адекватными для узких зон (см. рис. 2,b). Другой способ упрощения, который мы здесь не будем применять, состоит в замене функции $\Phi(k)$ ее средним по зоне Бриллюэна значением, равным 2 (приближение бездисперсионных зон [14]).

Представим $D_{ \pm}(\omega, k)$ в виде

$$
D_{ \pm}(\omega, k)=d_{ \pm(\omega)}-d(\omega) \cos (2 k a),
$$

где $\quad d_{ \pm}(\omega)=(\omega \pm t)\left[\omega\left(\omega-\varepsilon_{a}\right)-\tilde{t}^{2}\right]-d(\omega)$ $d(\omega)=2 t^{2}\left(\omega-\varepsilon_{a}\right)$. Вычислив интеграл (8) (см., например, [17]), с учетом (13) и (14) при $d_{ \pm}^{2}(\omega)>d^{2}(\omega)$ получим,

$$
\begin{aligned}
G_{j}(\omega) & =\frac{1}{2}\left[G_{j}^{+}+G_{j}^{-}(\omega)\right], \\
G_{j}^{ \pm}(\omega) & =\frac{c_{j}^{0}(\omega)}{\sqrt{d_{ \pm}^{2}(\omega)-d^{2}(\omega)}} .
\end{aligned}
$$



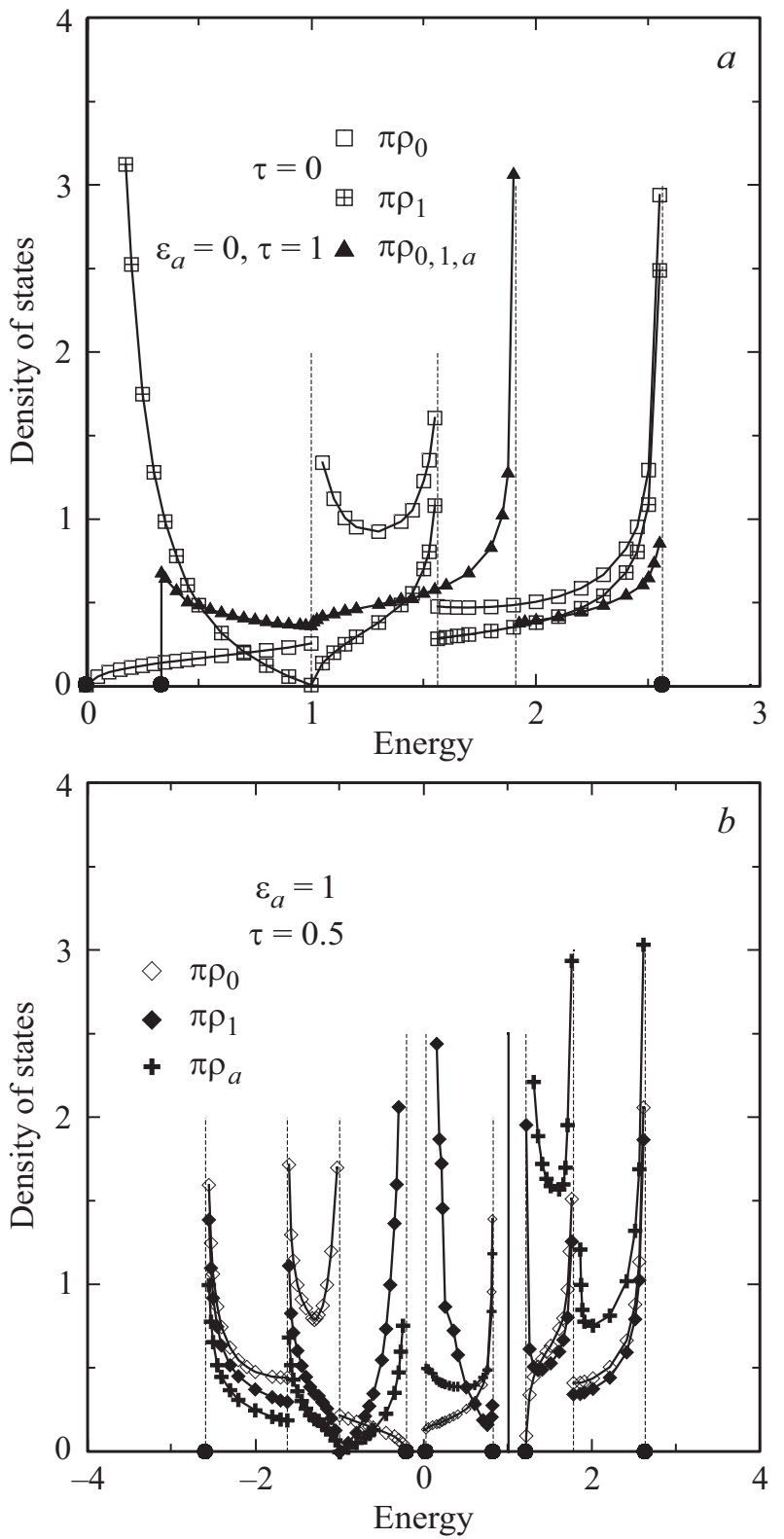

Рис. 3. Плотности состояний атомов углерода $\rho_{0}(\omega)$ и $\rho_{1}(\omega)$ и декорирующих частиц $\rho_{a}(\omega)$ в отсутствие подложки. Все энергетические величины измеряются в ед. $t$, плотности состояний - в ед. $\left.t^{-1} . a\right)$ Недекорированная $(\tau=0$, светлые пустые квадраты и квадраты с крестом) и декорированная $\left(\varepsilon_{a}=0\right.$, $\tau=1$, темные треугольники) наноленты. $b$ ) Декорированная нанолента при $\varepsilon_{a}=1$ и $\tau=0.5$. Штриховые линии соответствуют асимптотам (асимптота для $\rho_{1}(\omega)$ при $\tau=0$ (светлые квадраты с крестом на рис. $3, a)$ совпадает с осью абсцисс); жирная вертикальная линия на рис. $3, b$ изображает плотность состояний $\rho_{9}(\omega) \propto \delta(\omega-1)$, отвечающую бездисперсионной зоне (рис. 2,b).Темными кружками на оси энергии отмечены границы областей сплошного спектра.

При $\quad d_{ \pm}^{2}(\omega) \leq d^{2}(\omega) \quad$ нужно в $\quad(15)$ заменить радикал $\sqrt{d_{ \pm}^{2}(\omega)-d^{2}(\omega)}$ на $i \sqrt{d^{2}(\omega)-d_{ \pm}^{2}(\omega)}$. Так как $\rho_{j}(\omega)=-\pi^{-1} \Im G_{j}(\omega)$, то ясно, что сплошному спектру соответствуют области энергии, в которых $d_{ \pm}^{2}(\omega) \leq d^{2}(\omega)$. Таким образом, имеем

$$
\begin{gathered}
\rho_{j}(\omega)=\frac{1}{2}\left[\rho_{j 0}^{+}(\omega) \Theta_{+}+\rho_{j 0}^{-}(\omega) \Theta_{-}\right], \\
\rho_{j}^{ \pm}(\omega)=\frac{\left|c_{j}^{0}(\omega)\right|}{\pi \sqrt{d^{2}(\omega)-d_{ \pm}^{2}(\omega)}},
\end{gathered}
$$

где $\Theta_{ \pm} \equiv \Theta\left(|d(\omega)|-\left|d_{ \pm}(\omega)\right|\right)$ и $\Theta(\ldots)$ - функция Хэвисайда, причем нижний индекс 0 означает, что подложка отсутствует. Плотности состояний для тех же, что и на рис. 2, значений параметров $\varepsilon_{a}$ и $\tau$, представлены на рис. 3. Характерные расходимости функций $\rho_{j}(\omega)$ (рис. 3) связаны с квазиодномерностью задачи о краях наноленты (аналог одномерной модели Лаббе-Фриделя [18]). Для случая $\varepsilon_{a}=0$, $\tau=0$ (рис. 3, $a$, пустые квадраты и квадраты с крестом) имеем $c_{1}^{0}(\omega)=c_{a}^{0}(\omega)$; при $\varepsilon=0, \tau=1$ получаем $c_{0}(\omega)=c_{1}^{0}(\omega)=c_{a}^{0}(\omega)$ (рис. $3, a$, темные треугольники). Эти равенства являются результатом не только упрощений, связанных с переходом от коэффициентов $c_{1, a}$ к коэффициентам $c_{1, a}^{0}$, но и выбором параметров $\varepsilon_{a}$ и $\tau$. В общем случае, представленном на рис. $3, b$, имеем $c_{0}=\omega(\omega-t)-\ddot{t}^{2}, \quad c_{1}^{0}(\omega-t)\left(\omega^{2}-t^{2}\right) / \omega, \quad c_{a}^{0}=\omega^{2}-t^{2}$. Следствием равенства коэффициентов $c_{j}^{0}$ являются равенства соответствующих чисел заполнения $n_{j}$. Отметим, что с качественной точки зрения, приведенные здесь зависимости $\rho_{j}(\omega)$ аналогичны полученным в работах $[13,14]$. Отличием является конечные скачки функций $\rho_{0}(\omega)$ и $\rho_{1}(\omega)$, возникающие вследствие наличия экстремумов у зависимостей $\omega(k)$.

\section{4. Нанолента эпиграфена, декорированная адчастицами}

Как показано в работах [13,14], выражение (16) достаточно легко обобщить на случай слабой связи наноленты и декорирующих частиц с подложкой, когда отношения $\Gamma^{2} / t^{2}, \Gamma_{a}^{2} / t^{2}, \Gamma^{2} / \tilde{t}^{2}$ и $\Gamma_{a}^{2} / \tilde{t}^{2}$ можно считать малыми параметрами (режим слабой связи адсорбированного комплекса с субстратом). Тогда, по аналогии с $[13,14]$, вместо выражений (16) можно приближенно записать $\rho_{j}^{*}(\omega)=\sum_{\alpha} v_{j \alpha} \rho^{\alpha}(\omega)$, где $\rho^{\alpha}(\omega)=\Gamma / \pi\left[\left(\omega-\omega_{\alpha}\right)^{2}+\Gamma^{2}\right]$ и $v_{j \alpha}-$ весовые множители. Таким образом, в режиме слабой связи плотность состояний $\rho_{j}^{*}(\omega)$ представляет собой сумму лоренцовских контуров $\rho^{\alpha}(\omega)$ с весами $v_{j \alpha}$. При этом в точках расходимостей плотностей состояний $\rho_{j}(\omega)$ (рис. 3) у $\rho_{j}^{*}(\omega)$ появляются конечные максимумы с полушириной $\Gamma$ (на половине высоты), функции $\rho_{j}^{*}(\omega)$ размываются, оставаясь ненулевыми на всей энергетической оси. В этом и состоит основной эффект, вносимый металлической подложкой.

В настоящей работе мы, однако, подробно рассмотрим противоположную ситуацию - режим сильной 
связи с подложкой адсорбционного комплекса нанолента графена - декорирующие частицы. Полагая в (2)-(4) отношения $t^{2} / \Gamma^{2}, \tilde{t}^{2} / \Gamma^{2}, t^{2} / \Gamma_{a}^{2}, \tilde{t}^{2} / \Gamma_{a}^{2}$ малыми параметрами и проинтегрировав по зоне Бриллюэна, получим $G_{0}(\omega) \approx\left(1+3 g^{2} t^{2}\right), G_{1}(\omega) \approx g\left(1_{2} g^{2} t^{2}+g g_{a} \tilde{t}^{2}\right)$, $g_{a}(\omega) \approx g_{a}\left(1+g g_{a} \tilde{t}^{2}\right)$, или

$$
\begin{gathered}
\rho_{0}^{*}(\omega)=\bar{\rho}(\omega)\left(1+3 t^{2} \frac{3 \omega^{2}-\Gamma^{2}}{\left(\omega^{2}+\Gamma^{2}\right)^{2}}\right) \\
\rho_{1}^{*}(\omega)=\bar{\rho}(\omega)\left(1+2 t^{2} \frac{3 \omega^{2}-\Gamma^{2}}{\left(\omega^{2}+\Gamma^{2}\right)^{2}}\right. \\
\left.+\tilde{t}^{2} \frac{2 \omega\left(\omega-\varepsilon_{a}\right)+\gamma_{a} \omega^{2}-\Gamma \Gamma_{a}}{\left(\omega^{2}+\Gamma^{2}\right)\left[\left(\omega-\varepsilon_{a}\right)^{2}+\Gamma_{a}^{2}\right]}\right), \\
\rho_{a}^{*}(\omega)=\bar{\rho}_{a}(\omega)\left(1+\tilde{t}^{2} \frac{2 \omega\left(\omega-\varepsilon_{a}\right)+\gamma_{a}^{-1}\left(\omega-\varepsilon_{a}\right)^{2}-\Gamma \Gamma_{a}}{\left(\omega^{2}+\Gamma^{2}\right)\left[\left(\omega-\varepsilon_{a}\right)^{2}+\Gamma_{a}^{2}\right]}\right),
\end{gathered}
$$

где $\gamma_{a}=\Gamma_{a} / \Gamma$ и локальные плотности состояний на изолированных адатомах графена и адчастицах равны соответственно

$$
\bar{\rho}(\omega)=\frac{1}{\pi} \frac{\Gamma}{\omega^{2}+\Gamma^{2}}, \quad \bar{\rho}_{a}(\omega)=\frac{1}{\pi} \frac{\Gamma_{a}}{\left(\omega-\varepsilon_{a}\right)^{2}+\Gamma_{a}^{2}} .
$$

Легко показать, что при $\varepsilon_{a}=0$ плотности состояний представляют собой двугорбые кривые с экстремумами, равными $\rho_{0}^{*}(0)=\left(1-3 t^{2} / \Gamma^{2}\right) / \pi \Gamma, \rho_{1}^{*}(0)=\left(1-2 t^{2} / \Gamma^{2}-\right.$ $\left.-3 \tilde{t}^{2} / \Gamma \Gamma_{a}\right) / \pi \Gamma \quad$ и $\quad \rho_{a}^{*}(0)=\left(1-3 \tilde{t}^{2} / \Gamma \Gamma_{a}\right) / \pi \Gamma$. При $|\omega| \gg\left|\varepsilon_{a}\right|, \quad \Gamma$ имеем $\rho_{j}^{*}(\omega) \propto \Gamma / \pi \omega^{2}$. Из рис. 4, $a$ $\left(\Gamma=\Gamma_{a}=2 t, \tau=0.7, \varepsilon_{a}=0\right)$ следует, что исходные одногорбые лоренцевы распределения $\bar{\rho}(\omega)$ и $\bar{\rho}_{a}(\omega)$ преобразуются в двугорбые кривые $\rho_{0}^{*}(\omega)$ и $\rho_{1}^{*}(\omega)$, тогда как для $\rho_{a}^{*}(\omega)$ наблюдается лишь уплощение максимума. При $\varepsilon_{a}=0$ имеем $\rho_{j}^{*}(\omega)=\rho_{j}^{*}(-\omega)$. Если же $\varepsilon_{a} \neq 0$, эта симметрия нарушается, что и отражает рис. $4, b\left(\varepsilon_{a}=t\right)$. Так, например, при $\omega \rightarrow 0$ получаем

$$
\begin{aligned}
\rho_{1}^{*}(\omega) \approx & (\pi \Gamma)^{-1}\left[1-2 t^{2} / \Gamma^{2}-\gamma_{a} \tilde{t}^{2} /\left(\varepsilon_{a}^{2}+\Gamma_{a}^{2}\right)\right. \\
& \left.-2 \omega \varepsilon_{a} \tilde{t}^{2} / \Gamma^{2}\left(\varepsilon_{a}^{2}+\Gamma_{a}^{2}\right)\right] \\
\rho_{a}^{*}(\omega) \approx & \left(\pi \Gamma_{a}\right)^{-1}\left[1-\gamma_{a} \tilde{t}^{2} /\left(\varepsilon_{a}^{2}+\Gamma^{2}\right)\right. \\
& -2 \omega \varepsilon_{a}\left(1+\gamma_{a}^{-1}\right) \tilde{t}^{2} / \Gamma^{2}\left(\varepsilon_{a}^{2}+\Gamma_{a}^{2}\right) .
\end{aligned}
$$

Отметим, что плотности состояний $\rho_{1}^{*}(\omega)$ и $\rho_{a}^{*}(\omega)$ инвариантны относительно одновременной замены $\varepsilon_{a}$ на $-\varepsilon_{a}$ и $\omega$ на $-\omega$.

Перейдем теперь к расчету чисел заполнения $n_{j}^{*}$. Так как при нулевой температуре $n_{j}^{*}=2 \int_{-\infty}^{\varepsilon_{\mathrm{F}}} \rho_{j}^{*}(\omega) d \omega$, получим

$$
n_{0}^{*}=\bar{n}-\frac{6 t^{2} \Gamma \varepsilon_{\mathrm{F}}}{\pi\left(\varepsilon_{\mathrm{F}}^{2}+\Gamma^{2}\right)^{2}},
$$

где $\bar{n}=(2 / \pi) \operatorname{arcctg}\left(-\varepsilon_{\mathrm{F}} / \Gamma\right)-$ число заполнения изолированного адатома графена (см. [17]). Для вычисления $n_{1}^{*}$
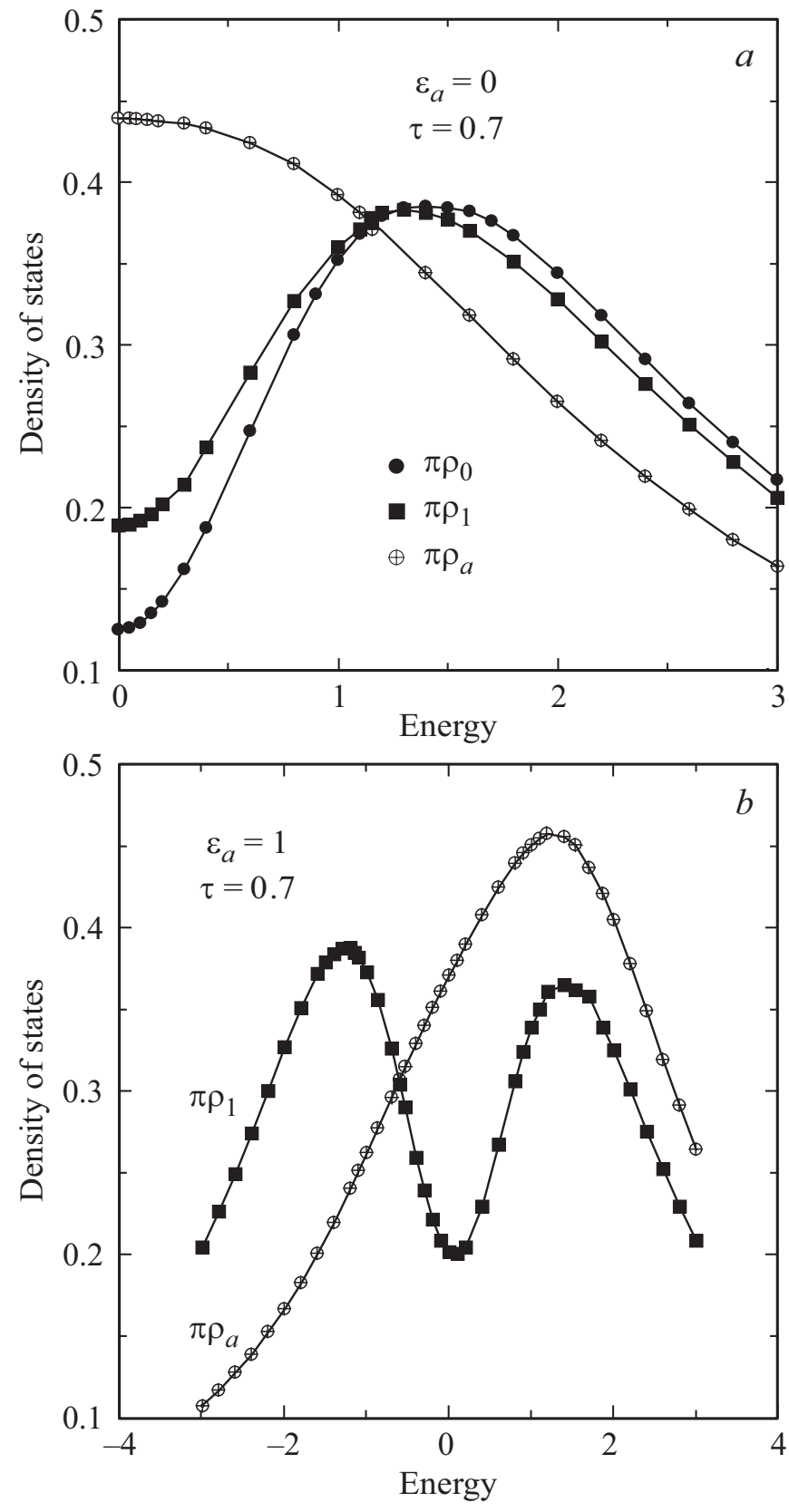

Рис. 4. Плотности состояний $\rho_{0}^{*}(\omega)$ (темные кружки), $\rho_{1}^{*}(\omega)$ (темные квадраты) и $\rho_{a}^{*}(\omega)$ (светлые кружки с крестом) в режиме сильной связи с подложкой при $\Gamma=\Gamma_{a}=2, \tau=0.7$ и $\varepsilon_{a}=0(a)$ и $1(b)$. Все энергетические величины измеряются в ед. t, плотности состояний -в ед. $t^{-1}$. На рис. $4, a$ изображены только правые (отвечающие положительной энергии) части кривых.

и $n_{a}^{*}$ положим $\Gamma_{a}=\Gamma$. Тогда, пренебрегая для простоты членами порядка $\varepsilon_{a}^{2} / \Gamma^{2}$, найдем

$$
\begin{gathered}
n_{1}^{*}=\bar{n}-4 t^{2} \Gamma \varepsilon_{\mathrm{F}} / \pi\left(\varepsilon_{\mathrm{F}}^{2}+\Gamma^{2}\right)^{2}+2 \pi^{-1} \tilde{t}^{2} \Gamma(A+B) \\
n_{a}^{*}=\bar{n}_{a}+2 \pi^{-1} \tilde{t}^{2} \Gamma(A-B)
\end{gathered}
$$




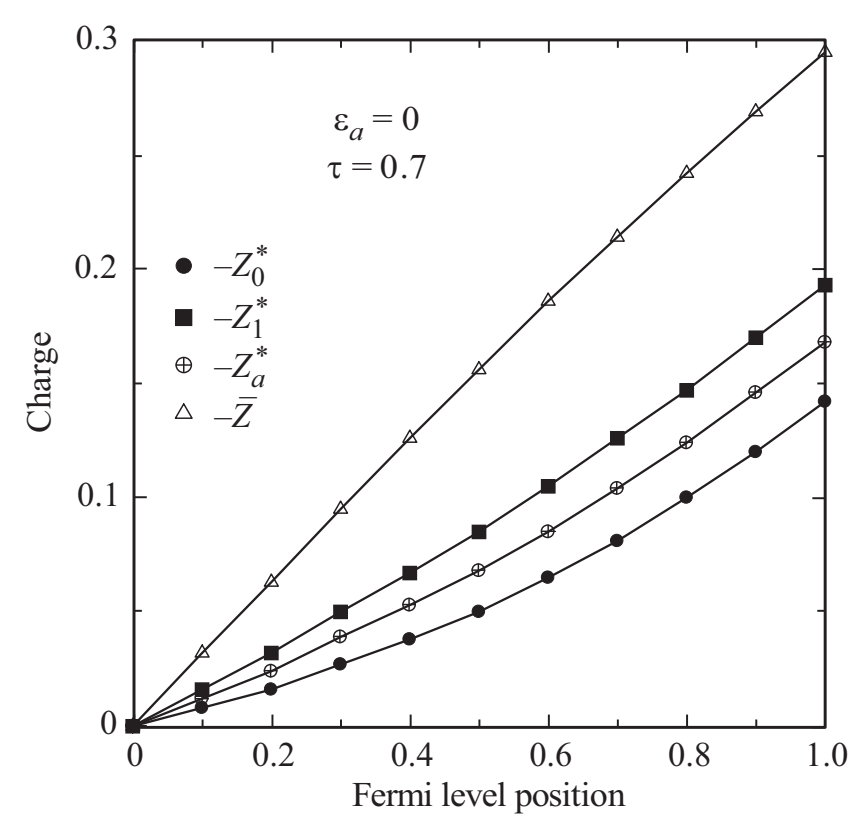

Рис. 5. Зависимости зарядов адатомов графена $Z_{0}^{*}$ (темные кружки) и $Z_{1}^{*}$ (темные квадраты), декорирующих адчастиц $Z_{a}^{*}$ (светлые кружки с крестами) и изолированных адатома углерода и адчастицы $\bar{Z}=\bar{Z}_{a}$ (светлые ромбы) от положения уровня Ферми $\varepsilon_{\mathrm{F}}>0$ при $\Gamma=\Gamma_{a}=2, \tau=0.7$ и $\varepsilon_{a}=0$. На рис. 5 область энергий $\varepsilon_{\mathrm{F}}<0$ не отображена, так как $\left|Z_{j}^{*}\left(\varepsilon_{\mathrm{F}}\right)\right|=\left|Z_{j}^{*}\left(-\varepsilon_{\mathrm{F}}\right)\right|$ и $\left.\left|\bar{Z}_{j}\left(\varepsilon_{\mathrm{F}}\right)\right|=\left|\bar{Z}_{j}\left(-\varepsilon_{\mathrm{F}}\right)\right|\right)$. Энергия уровня Ферми измеряется в ед.t.

где $\bar{n}=(2 / \pi) \operatorname{arcctg}\left(-\varepsilon_{\mathrm{F}} / \Gamma\right)-$ число заполнения изолированной адчастицы и

$$
\begin{gathered}
A=-\frac{\varepsilon_{\mathrm{F}}}{\left(\varepsilon^{2}+\Gamma^{2}\right)^{2}}-\frac{\varepsilon_{a}\left(3 \varepsilon_{\mathrm{F}}^{2}-\Gamma^{2}\right)}{2\left(\varepsilon^{2}+\Gamma^{2}\right)^{3}}, \\
B=\frac{\varepsilon_{a}\left(3 \varepsilon_{\mathrm{F}}^{2}-\Gamma^{2}\right)}{\left(\varepsilon^{2}+\Gamma^{2}\right)^{3}} .
\end{gathered}
$$

На рис. 5 представлены зависимости зарядов адатомов графена и декорирующих адчастиц $Z_{i j}^{*}=1-n_{j}^{*}$, а также изолированных (одиночных) адатомов углерода и адчастиц $\bar{Z}=1-\bar{n}=\bar{Z}_{a}=1-\bar{n}_{a}$ от энергии уровня Ферми $\varepsilon_{\mathrm{F}}$ для $\Gamma=\Gamma_{a}=2 t, \tau=0.7$ и $\varepsilon_{a}=0$. При $\varepsilon_{\mathrm{F}}>0$ все заряды отрицательны, при $\varepsilon_{\mathrm{F}}<0$ положительны, причем, $\left|Z_{j}^{*}\left(\varepsilon_{\mathrm{F}}\right)\right|=\left|Z_{j}^{*}\left(-\varepsilon_{\mathrm{F}}\right)\right|$ и $\left|\bar{Z}\left(\varepsilon_{\mathrm{F}}\right)\right|=\left|\bar{Z}\left(\varepsilon_{\mathrm{F}}\right)\right|$. Из рис. 5 следует, что $|\bar{Z}|>\left|Z_{1}^{*}\right|>\left|Z_{a}^{*}\right|>\left|Z_{0}^{*}\right|$ при $\varepsilon_{\mathrm{F}} \neq 0$. Этот результат в корне отличается от случая свободной наноленты с декорирующими частицами, где, согласно предложенной нами схеме оценок, все числа заполнения равны 1, а заряды - 0 . Величина заряда численно равна значению перехода заряда между адсорбатом и субстратом. При положительном заряде адсорбата имеет место переход электронов с адсорбата в подложку, при отрицательном - из подложки на адсорбат. Неравенства $|\bar{Z}|>\left|Z_{0,1}^{*}\right|$ и $\left|\bar{Z}_{a}\right|>\left|Z_{a}^{*}\right|$ показывают, что учет взаимодействия адатомов углерода между собой и с адчастицами понижает величины всех зарядов, т.е. ведет к деполяризации адсорбата. Этот результат хорошо известен в теории адсорбции $[15,16]$.

\section{5. Декорирование цепочки адатомов углерода}

Одномерные углеродные структуры (карбины) вызывают интерес уже достаточно долгое время (см., например, [19-22] и ссылки, приведенные там), но некоторые связанные с ними вопросы так и остаются открытыми. Различают два вида карбинов: металлический кумулен с двойными связями $(\ldots=C=C=\ldots)$ и полупроводниковый полиин с чередующимися одинарными и тройными связями $(\ldots-C \equiv C-\ldots)$, между которыми возможен пайерлсовский переход. В рамках постановки рассматриваемой здесь проблемы, карбин интересен тем, что, активно присоединяет посторонние молекулы (в частности, ДНК [22]) и может выступать в качестве сенсора. Таким образом, проблема декорирования является для карбина актуальной. Здесь мы рассмотрим кумулен, характеризуемый однородным распределением $\pi$-электронов вдоль цепочки [19].

Воспользуемся результатами работы [13], заменив на рис. 1 этой работы адатомы графена типа $-2-1$, 0-1 и 2-1 декорирующими адчастицами. Такое расположение декорирующих частиц имеет вполне определенную аналогию с графаном, где атомы водорода расположены по обеим сторонам графенового листа, что уменьшает кулоновское межатомное отталкивание их электронов. Таким образом, имеем симметричную структуру, представляющую собой цепочку адатомов углерода (эпикарбин) с прикрепленными к ней с двух сторон адчастицами. Тогда для углеродных адатомов и адчастиц из выражений (4) и (5) из [13] получим соответственно следующие функции Грина:

$$
\begin{aligned}
& G^{\prime}(\omega, k) \equiv G_{0}^{\prime}(\omega, k)=G_{1}^{\prime}(\omega, k) \\
&=g\left(1-g g_{a} \tilde{t}^{2}-\frac{g^{2} t^{2} \Phi(k)}{1-g g_{a} \tilde{t}^{2}}\right)^{-1}, \\
& G_{a}^{\prime}(\omega, k)=g_{a}\left(1-\frac{g g_{a} \tilde{t}^{2}}{1-\frac{g^{2} t^{2} \Phi(k)}{1-g g_{a} \tilde{t}^{2}}}\right)^{-1},
\end{aligned}
$$

где $|k| \leq \pi / 2 a$. Полюса функций Грина (26) и (27), определяющие закон дисперсии, являются корнями уравнения

$$
\left(\omega-\varepsilon_{a}\right)\left[\omega^{2}-t^{2} \Phi(k)\right]-2 \omega \tilde{t}^{2}=0 .
$$

Соответствующие зоны изображены на рис. 6 для различных значений параметров задачи. При $k=\pi / 2 a$ имеем $\omega_{0}=0$ и $\omega_{ \pm}(\pi / 2 a)=\left(\varepsilon_{a} \pm \sqrt{\varepsilon_{a}^{2}+8 \tilde{t}^{2}}\right) / 2$. Если $\varepsilon_{0}=0$, то $\omega_{0}=0$ и $\omega_{ \pm(k)} \pm t \sqrt{\Phi(k)+2 \tau^{2}}$. В общем случае $\left(\varepsilon_{a}=t, \tau=0.5\right)$ симметрия зон, отвечающих положительным и отрицательным энергиям нарушается. 


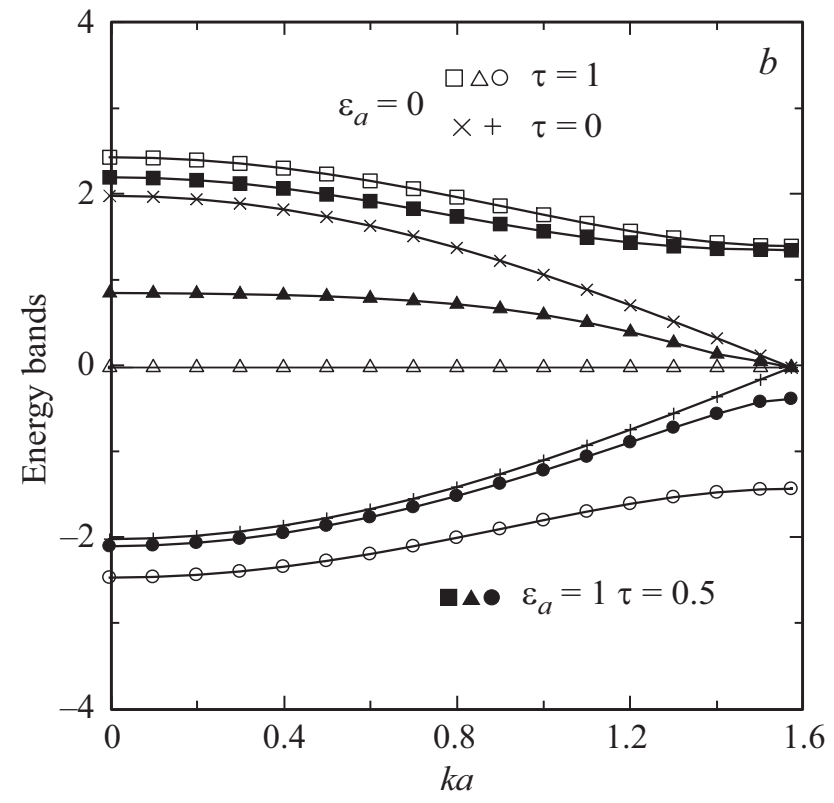

Рис. 6. Энергетические зоны декорированной цепочки атомов углерода для $\varepsilon_{a}=0$ (светлые символы) и $\varepsilon_{a}=1$ (темные символы) при $\tau=0.5$ (темные квадраты, треугольники и кружки) и $\tau=1$ (светлые квадраты, треугольники и кружки). Энергетическая зона недекорированной цепочки $(\tau=0$, прямые и косые кресты) сведена в зону Бриллюэна декорированной цепочки. Энергии зон приведены в ед.t.

Сравнение рис. 6 с рис. 2 показывает качественное согласие спектров за тем, однако, важным исключением, что в случае цепочки на зависимостях $\omega(k)$ отсутствуют экстремумы в интервале $0<|k|<\pi / 2 a$, наблюдаемые для наноленты. Ниже, как и в п. 4, мы рассмотрим режим сильной связи декорированной углеродной цепочки и адчастиц с металлической подложкой, что может быть определено как декорированный эпикарбин.

Полагая в (27) и (28) отношения $t^{2} / \Gamma^{2}, \tilde{t}^{2} / \Gamma^{2}$, $t^{2} / \Gamma_{a}^{2}, \tilde{t}^{2} / \Gamma_{a}^{2}$ малыми параметрами и проинтегрировав по зоне Бриллюэна, получим функции Грина $g^{\prime}(\omega) \approx g\left(1+2 g^{2} t^{2}+g g_{a} \tilde{t}^{2}\right), G_{a}^{\prime}(\omega) \approx g_{a}\left(1+g g_{a} \tilde{t}^{2}\right)$, идентичные функциям Грина $G_{1}(\omega)$ и $G_{a}(\omega)$ из п. 4. Отсюда следует, что формулы (18), (19), (24) и (25) справедливы и для декорированной углеродной цепочки. Справедливы и результаты, представленные на рис. 4 и 5: $\rho_{a}(\omega)$ соответствует $\rho^{\prime}(\omega), \rho_{a}(\omega)-\rho_{a}^{\prime}(\omega)$, $Z_{1}^{*}-Z^{\prime}$ и $Z_{a}^{*}-Z_{a}^{\prime}$. Таким образом, в приближении сильной связи с подложкой задача о декорированном эпикарбине сводится к задаче о декорированной наноленте эпиграфена.

\section{6. Выводы}

1. Сопоставление результатов зонных расчетов в рамках использованных в настоящей работе двухцепочечной и одноцепочечной моделей показывает, помимо прочего, что лишь первая модель правильно отражает электронную структуру наноленты графена, то есть наличие экстремумов у зависимостей $\omega(k)$. С другой стороны, вторая модель представляется вполне приемлемой для описания металлического карбина.

2. Из сопоставления полученных здесь зависимостей зарядов адатомов углерода и адчастиц от положения уровня Ферми в режиме сильной связи с подложкой, с соответствующими зависимостями, полученными в [14] в задаче о декорировании кромки графена в режиме слабой связи, следует, что эти зависимости качественно согласуются. Это согласие подтверждает важный результат теории адсорбции [15]: оценить величину чисел заполнения для адатомов, образующих когерентные решетки, можно в модели адсорбированного димера. Отметим, что приближенные значения функций Грина $G_{0}(\omega), G_{1}(\omega), G_{a}^{\omega}$ (п. 4) и $G^{\prime}(\omega), G_{a}^{\prime}(\omega)$ (п. 5) для режима сильной связи отвечают именно адсорбированным димерам.

Оба приведенных вывода серьезно упрощают оценки. Численные расчеты при почти полном отсутствии экспериментальной информации представляется нам преждевременным.

\section{Список литературы}

[1] R. Taira, A. Yamanaka, S. Okada. Appl. Phys. Express 9, 115102 (2016).

[2] M.R. Mananghaya, G.N. Santos, D. Yu, C. Stampfl. Sci. Rep. 7, 15727 (2017)

[3] K. Nakada, M. Fujita, G. Dresselhaus, M. Dresselhaus. Phys. Rev. B 54, 17954 (1996).

[4] M. Fujita, K. Wakabayashi, K. Nakada, K. Kusakabe. J. Phys. Soc. Jpn 65, 1920 (1996).

[5] Y. Miyamoto, K. Nakada, M. Fujita. Phys. Rev. B 59, 9858 (1999).

[6] S. Okada, M. Igami, K. Nakada, A. Oshiyama. Phys. Rev. B 62, 9896 (2000).

[7] M.P. Levendorf, C.-J. Kim, L. Brown, P.Y. Huang, R.W. Havener, D.A. Muller, J. Park. Nature 488, 627 (2012).

[8] Z. Liu, L. Ma, G. Shi, W. Zhou, Y. Gong, S. Lei, X. Yang, J. Zhang, J. Yu, K.P. Hackenberg, A. Babakhani, J.C. Idrobo, R. Vajtai, J. Lou, P.M. Ajayan. Nature Nanotechnology 8, 119 (2013).

[9] G.C. Loh, R. Pandey. J. Mater. Chem. C 3, 5918 (2015).

[10] С.Ю. Давыдов. ФТТ 60, 1389 (2018).

[11] J. Cserti. Am. J. Phys. 68, 896 (2000).

[12] G. Jose, R. Malla, V. Srinivasan, A. Sharma, S. Gangadharaiah. arXiv: $1711.08204 \mathrm{v} .1$.

[13] С.Ю, Давыдов. Цепочечная модель декорирования зигзагообразной кромки графена. ФТП 53, 83 (2019).

[14] С.Ю. Давыдов. Письма в ЖТФ 44 (21), 55 (2018).

[15] С.Ю. Давыдов. Теория адсорбиии: метод модельных гамильтонианов. Изд-во СПбГЭТУ „ЛЭТИ“, СПб. (2013). 235 c. twirpx.com/file/1596114/

[16] С.Ю. Давыдов, А.А. Лебедев, О.В. Посредник. Элементарное введение в теорию наносистем. Лань, СПб. (2014). 192 c.

[17] Г.Б. Двайт. Таблицы интегралов и другие математические формулы. Лань, СПб. (2009). 232 с. 
[18] С.В. Вонсовский, Ю.А. Изюмов, Э.3. Курмаев. Сверхпроводимость переходных металлов, их сплавов $u$ соединений. Наука, М. (1977). Гл. V. 384 с.

[19] F. Banhart. J. Nanotechnology, 6, 559 (2015).

[20] L. Shi, P. Rohringer, K. Suenaga, Y. Niimi, J. Kotakoski, J.C. Meyer, H. Peterlik, M. Wanko, S. Cahangirov, A. Rubio, Z.J. Lapin, L. Novotny, P. Ayala, T. Pichler. Nature Mater. 15, 634 (2016).

[21] L. Shi, P. Rohringer, M. Wanko, A. Rubio, S. Waserroth, S. Reich, S. Cambre, W. Wenseleers, P. Ayala, T. Pichler. Phys. Rev. Mater. 1, 075601 (2017).

[22] Z. Salman, A. Nair, S. Tung. Proc. 12th IEEE Int. Conf. on Nano/micro Engineered and Molecular Systems (April 9-12, 2017), Los Angeles, USA. P. 667-681.

Редактор К.В. Емиев 